\title{
DETECTION OF CARRIERS
}

\author{
Helen M Kingston
}

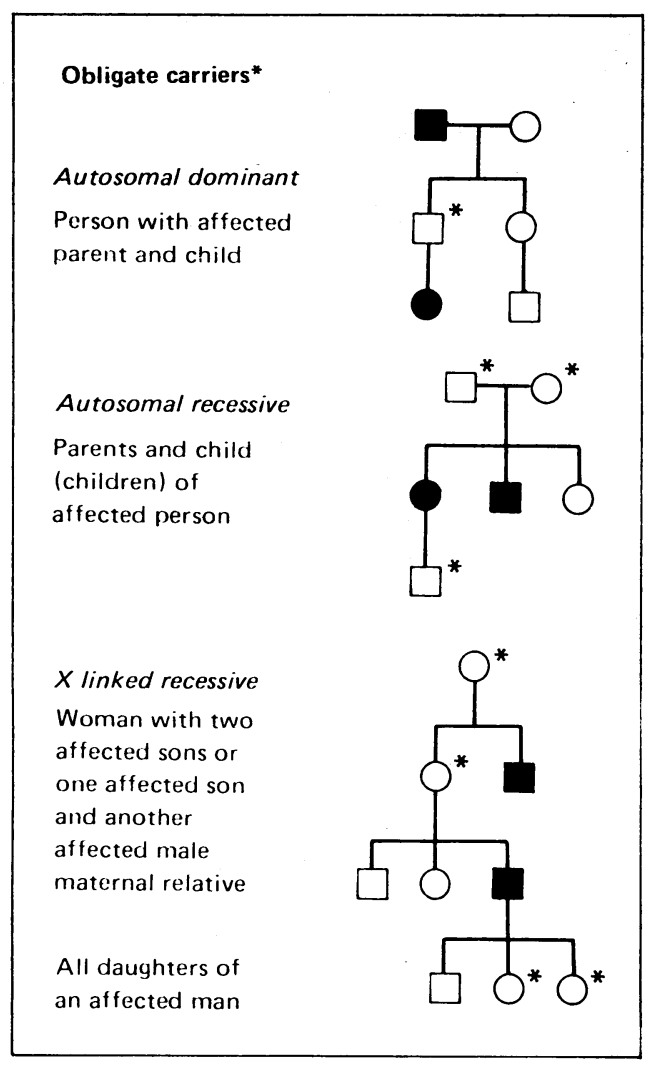

\section{Autosomal dominant disorders}

\section{Some autosomal dominant disorders amenable to carrier detection}

Adult polycystic kidney disease

Familial hypercholesterolaemia

Huntington's chorea

Malignant hyperpyrexia

Myotonic dystrophy

Neurofibromatosis

Tuberous sclerosis

von Hippel-Lindau disease
Identifying carriers of genetic disorders in families or populations at risk plays an important part in preventing genetic disease. A carrier is a healthy person who possesses the mutant gene for an inherited disorder in the heterozygous state. The term carrier is therefore restricted to people at risk of transmitting mendelian disorders and does not apply to parents whose children have chromosomal abnormalities or congenital malformations such as neural tube defect. An exception is that people who have balanced chromosomal translocations are referred to as carriers as the inheritance of balanced or unbalanced translocations follows mendelian principles.

In families in which there is a genetic disorder some members must be carriers because of the way in which the condition is inherited. These obligate carriers can be identified by drawing a family pedigree and do not require testing as their genetic state is not in doubt. Identification of obligate carriers is important not only for their counselling but also for defining a group in whom tests for carrier state can be evaluated.

Knowledge is needed of the proportion of obligate carriers showing abnormalities on clinical examination or with specific investigations and of the age at which such abnormalities appear to assess the likelihood of carrier state in the other relatives.

In some disorders - for example, sickle cell disease - all carriers can be identified with certainty; in others - for example, tuberous sclerosis - only a proportion can be identified. In autosomal dominant and X linked recessive disorders parental carrier state may be particularly difficult to assess because of the additional possibilities of new mutation in the child or germline mosaicism in the parent.

In autosomal dominant conditions most heterozygous subjects are clinically affected and testing for carrier state applies only to disorders that either are variable in their manifestations or have a late onset. Gene carriers in conditions such as tuberous sclerosis may be mildly affected but run the risk of having severely affected children whereas carriers in other disorders, such as Huntington's chorea, are destined to develop severe disease themselves.

Identifying symptomless gene carriers allows a couple to make informed decisions about having children, may indicate a need to avoid environmental triggers (as in porphyria), or may permit early treatment and prevention of complications (for example, in von Hippel-Lindau disease). Although testing for carrier state can have important benefits in conditions in which the prognosis is improved by early detection, presymptomatic diagnosis of severe disorders, such as Huntington's chorea, that are not amenable to treatment presents problems. Exclusion of carrier state is, however, equally important, removing anxiety about transmitting the condition to offspring and the need for long term follow up. 
Important autosomal recessive disorders amenable to carrier detection

Population based screening

Thalassaemia

Tay-Sachs disease

Sickle cell disease

\section{Family based testing *}

a-1-Antitrypsin deficiency

Congenital adrenal hyperplasia

Cystic fibrosis

Galactosaemia

Mucopolysaccharidosis I (Hurler's syndrome)

Phenylketonuria

* Indicated or feasible in families with an affected member
In autosomal recessive conditions carriers remain healthy. Occasionally, heterozygous subjects may show minor abnormalities, such as altered red cell morphology in sickle cell disease and mild anaemia in thalassaemia. Most inborn errors of metabolism follow autosomal recessive inheritance, and heterozygous subjects may show reduced activities of specific enzymes, which provides the basis for detecting carriers.

The parents of an affected child are obligate carriers, but testing may be appropriate for the healthy siblings of an affected person and their partner if the condition is fairly common. Testing may also be important for consanguineous couples with a positive family history of genetic disease. The main opportunity for preventing autosomal recessive disorders, however, depends on population screening programmes, which will identify couples at risk before the birth of an affected child within the family. Screening subgroups of the population at high risk has proved effective in Tay-Sachs disease and $\beta$ thalassaemia and will be appropriate for cystic fibrosis once a reliable test is available.

\section{$\mathrm{X}$ linked recessive disorders}

\author{
Some $X$ linked recessive disorders amenable \\ to carrier detection \\ Albinism (ocular) \\ Angiokeratoma (Fabry's disease) \\ Chronic granulomatous disease \\ Ectodermal dysplasia (anhidrotic) \\ Fragile $X$ syndrome \\ Haemophilia A \\ Haemophilia B \\ Ichthyosis (steroid sulphatase deficiency) \\ Lesch-Nyhan syndrome \\ Menkes's syndrome \\ Mucopolysaccharidosis II (Hunter's syndrome) \\ Muscular dystrophy (Duchenne and Becker's) \\ Ornithine transcarbamylase deficiency \\ Retinitis pigmentosa
}

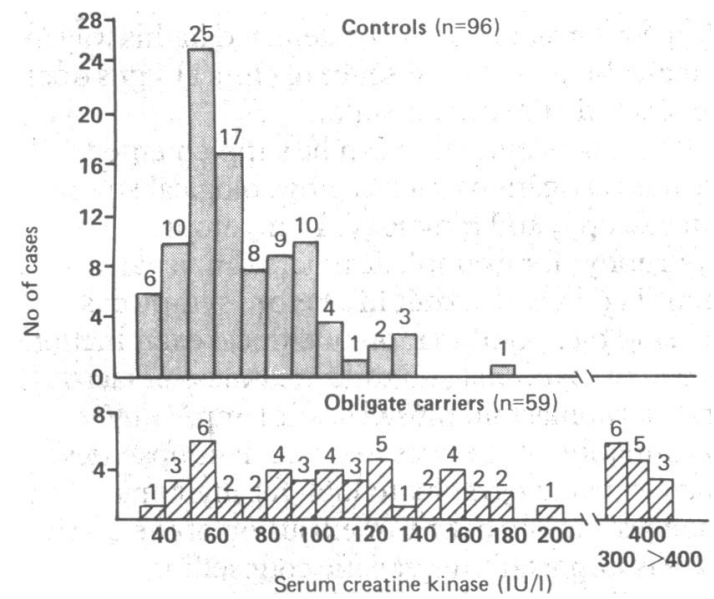

Overlapping ranges of serum creatine kinase activity in controls and obligate carriers of Becker's muscular dystrophy. (Ranges vary among laboratories.)
Carrier detection in $\mathrm{X}$ linked recessive conditions is particularly important as these disorders are often severe and in an affected family many female relatives may be at risk of having affected sons, irrespective of whom they marry. Genetic counselling cannot be undertaken without accurate assessment of carrier state, and calculating the risk is often complex.

Obligate carriers do not always show abnormalities on biochemical testing because of lyonisation, a process by which one or other $\mathrm{X}$ chromosome in female embryos is randomly inactivated early in embryogenesis. The proportion of cells with the normal or mutant $\mathrm{X}$ chromosome remaining active varies and will influence detection of carrier state. Carriers with a high proportion of normal X chromosomes remaining active will show no abnormalities on biochemical testing. Conversely, carriers with a high proportion of mutant $\mathrm{X}$ chromosomes remaining active are more likely to show biochemical abnormalities and may occasionally develop signs and symptoms of the disorder. Females with symptoms are called manifesting carriers.

Biochemical tests designed to determine carrier state must be evaluated initially in obligate carriers identified from affected families. Only tests which give significantly different results in obligate carriers compared with controls will be useful in determining the genetic state of female subjects at risk. Because the ranges of values in obligate carriers and controls overlap considerably (for example serum creatine kinase activity in X linked muscular dystrophy) the results for possible carriers are expressed in relative terms as a likelihood ratio. With this type of test confirmation of carrier state is always easier than exclusion. In muscular dystrophy a high serum creatine kinase activity confirms the carrier state; a normal result reduces but does not eliminate the chance that a female is a carrier. 

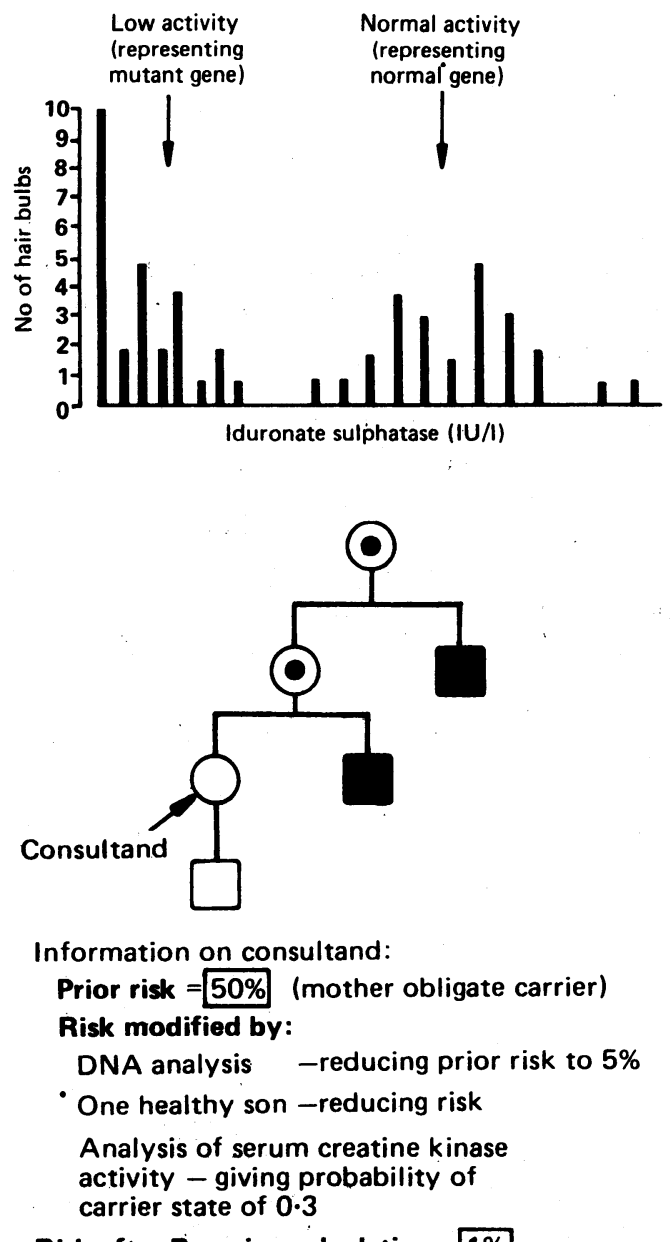

Risk after Bayesian calculation $=1 \%$
The problem of lyonisation can be largely overcome if biochemical tests can be performed on clonally derived cells; hair bulbs have been successfully used to detect carriers of Hunter's syndrome (mucopolysaccharidosis II). Carriers can be identified because they have two populations of hair bulbs, one with normal iduronate sulphatase activity, reflecting hair bulbs with the normal X chromosome remaining active, and the other with low enzyme activity, representing those with the mutant $\mathrm{X}$ chromosome remaining active.

Two populations of hair bulbs with low and normal activity of iduronate sulphatase, respectively, in female carrier of Hunter's syndrome.
DNA analysis is not affected by lyonisation and is becoming an increasingly important method of detecting carriers as specific gene probes or linked probes become available. Such analysis is successful in defining low as well as high risk.

Calculation of the final probability of carrier state entails analysis of pedigree data with the results of one or more specific tests. The possibility of new mutation and gonadal mosaicism must be taken into account in sporadic cases. The calculation relies on Bayesian analysis, and computer programs are available for the complex analysis required in large families.

Calculation of carrier risk in Duchenne muscular dystrophy.

\section{Testing for carrier state}

Various methods can be used to determine carrier state; those related directly to gene function discriminate better than those measuring functions further removed from the primary gene defect. Detection of an abnormality confirms the carrier state but its apparent absence does not guarantee normality.

\section{Clinical signs}

Careful examination for clinical signs may identify some carriers and is particularly important in autosomal dominant conditions in which the underlying biochemical basis of the disorder is unknown. In some $X$ linked recessive disorders (especially those affecting the eye or skin) abnormalities may be detected in this way in female carriers. The absence of clinical signs does not exclude the carrier state.

Clinical examination can be supplemented with investigations such as physiological studies, microscopy, and radiology. In myotonic dystrophy, for example, carriers can usually be identified in early adult life before symptoms develop by a combination of clinical examination to detect myotonia and mild weakness of facial and sternomastoid muscles, slit lamp examination of the eyes to detect lens opacities, and electromyography to look for myotonic changes. Confirmation or exclusion of the carrier state is important for genetic counselling, especially for mildly affected women who have an appreciable risk of producing severely affected infants with the congenital form of myotonic dystrophy. 


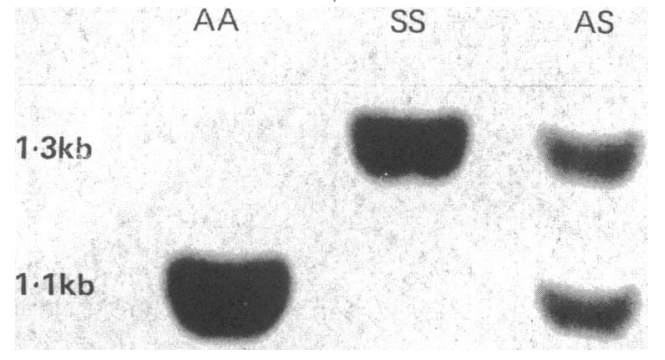

Identification of sickle cell carriers by Southern blot analysis of Mst II restriction fragments and $\beta$ globin gene probe. $(A A=$ Normal, $A S=$ sickle cell trait, $S S=$ sickle cell anaemia.)

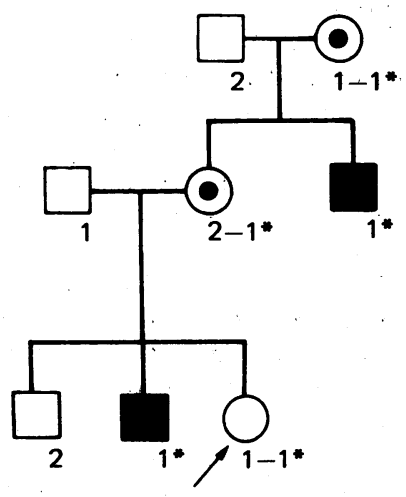

1,2 = DNA variants detected by probe linked to Duchenne muscular dystrophy gene on $X$ chromosome

Prediction of carrier state by DNA analysis in Duchenne muscular. dystrophy. Disease gene segregates with DNA variant $1 *$, predicting that consultand $(\lambda)$ is a carrier.

\section{Analysis of genes}

DNA probes are becoming available for an increasing number of mendelian disorders and can be used to predict carrier state as well as for presymptomatic or prenatal diagnosis. Specific gene probes may sometimes permit direct identification of carriers (as in sickle cell disease), but family studies are usually required to track the abnormal gene through the family with linked DNA polymorphisms (variations).

\section{Analysis of gene products}

Biochemical identification of carriers may be possible when the gene product is known. This approach is used for inborn errors of metabolism due to enzyme deficiency as well as for disorders due to a defective structural protein, such as haemophilia and thalassaemia. Overlap between the ranges of values in heterozygous and normal people occurs even when the primary gene product can be analysed, and interpretation of results can be difficult.

\section{Secondary biochemical abnormalities}

When the gene product is not known or cannot be readily tested the identification of carriers may depend on detecting secondary biochemical abnormalities, such as raised serum creatine kinase activity in Duchenne and Becker's muscular dystrophies. The overlap between the ranges of values in normal subjects and carriers is often considerable, and the sensitivity of this type of test is only moderate.

Dr Helen M Kingston, MD, is consultant clinical geneticist at St Mary's Hospital, Manchester.

The illustration of lens opacities was reproduced by kind permission of Professor P Harper, Institute of Medical Genetics for Wales, Cardiff.

\section{MATERIA NON MEDICA}

\section{Fretful porpentine?}

There is a long avenue lined by jacarandas that leads up to our cottage hospital gates. In spring they leave a carpet of mauve flowers on the ground. Last week I was driving out of the hospital down the avenue after a late night call when my headlights suddenly picked up an animal moving across the narrow tarmac. It was a porcupine. In fact, a baby hardly bigger than a hedgehog. It stopped still in its tracks and then proceeded to stare. I sat behind the wheel and waited. It seemed transfixed by the headlights so I gently switched them off. It sat down. I flashed the lights. There was still no action so I slowly eased myself out of the car in my best highway patrol manner.

Although it was small, I had heard that if they were angry these things could shoot their quills at you. You never know whether these stories about Africa are true, do you? It is because of such stories that I never go near swans in case I get my arm broken so I was not about to test the porcupine hypothesis in darkest night in darkest Africa. I talked to it in my therapeutic voice. A voice that has calmed a thousand brows. I appealed to its reason. It was small and I was big and other certain facts about life in the fast lane. It had no effect. It just looked at me with sad watery eyes. I then changed tactics to my authoritarian voice-absolutely no response. Just those offended penetrating eyes.

I now decided on action and walked to the side of the road to look for a stick with which I could gently push it off the road. The only one I could find was an intimidating four foot branch. I advanced slowly forward shielding my eyes against an anticipated avalanche of flying quills the like of which would not have been seen since Agincourt.

Cautiously I leaned forward and touched it with the end of the branch. It immediately bristled up in what I thought was an unnecessarily menacing and truculent manner. It was at this point that another car arrived. The driver got out and solicitously inquired what exactly his intrepid local GP was doing with that large branch he was hiding behind his back. I started to explain what I was about when he bent to pick the little chap up. I warned him it was in ugly mood.

"Yes," he said, as he picked it up in his hands and put it on the grass verge, "hedgehogs can be very dangerous."

CHRISTOPHER G ELLIS, general practitioner, Cullinan, South Africa 